\title{
Demonstration of Atmospheric-Pressure Radiometer with Metamaterial Vanes
}

Zhipeng Lu ${ }^{1}$, Mohsen Azadi' ${ }^{2}$, George A. Popov ${ }^{2}$, Christopher Stanczak ${ }^{2}$, Pratik Ponnarassery ${ }^{2}$, Andy G. Eskenazi ${ }^{2}$, John Cortes ${ }^{3}$, Matthew F. Campbell ${ }^{2}$, and Igor Bargatin ${ }^{2 *}$

${ }^{1}$ Department of Chemistry, University of Pennsylvania, USA;

${ }^{2}$ Department of Mechanical Engineering and Applied Mechanics, University of Pennsylvania, USA;

${ }^{3}$ Lawrence Livermore National Laboratory, USA.

* Corresponding author: bargatin@seas.upenn.edu 


\section{Demonstration of Atmospheric-Pressure Radiometer with Metamaterial Vanes}

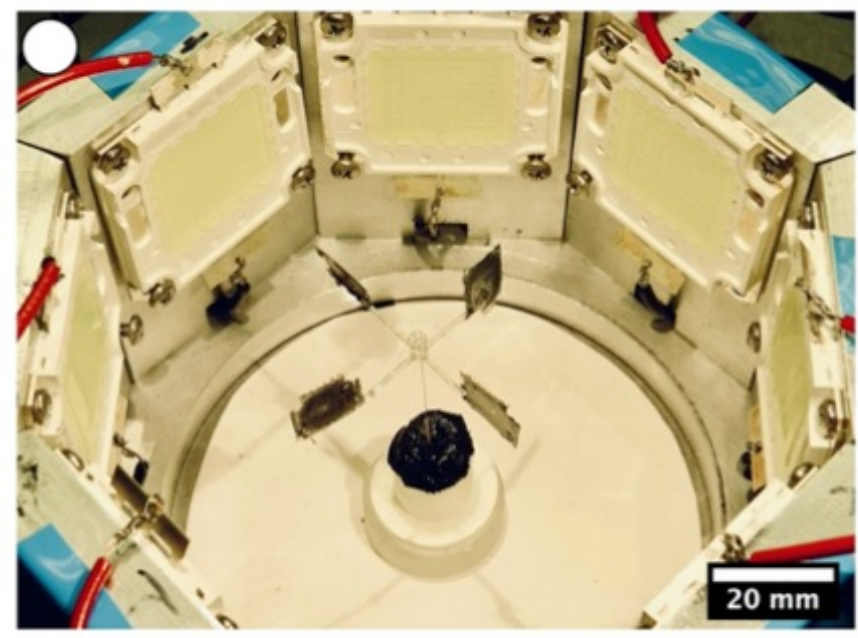

Photograph of radiometer inside test chamber.

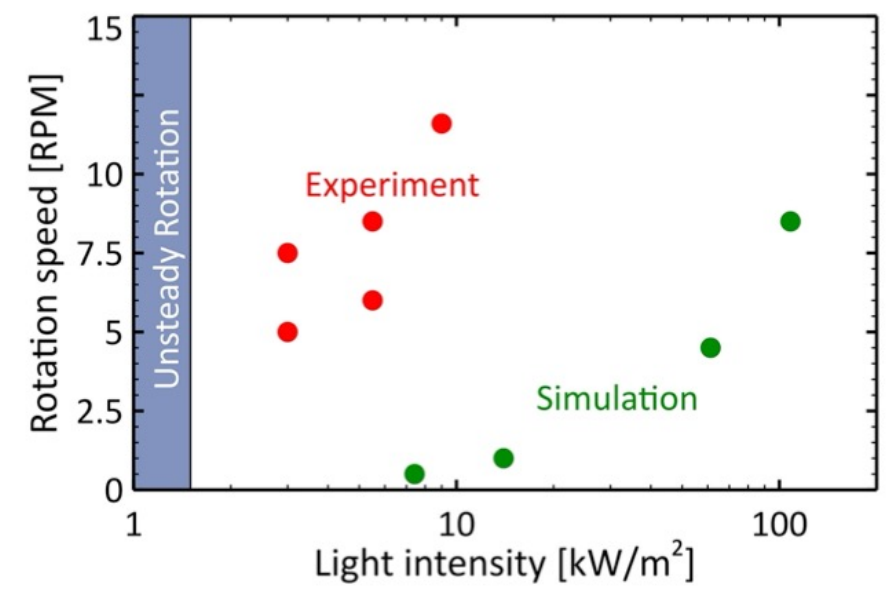

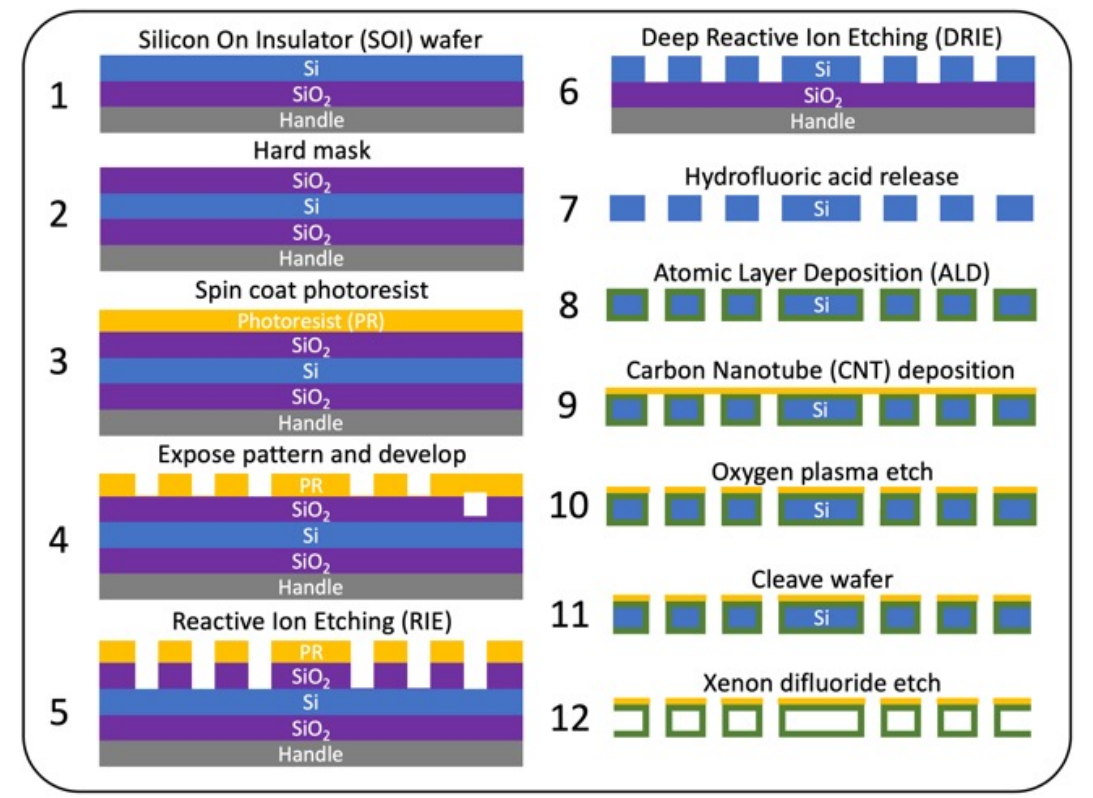

Vane fabrication process.

Experimentally-measured and numerically-calculated rotation speeds as a function of light intensity incident on the vanes. 
Abstract: We report a Crookes radiometer that rotates at atmospheric pressure using architected microporous dielectric plates, known as nanocardboard, as vanes [1,2]. The functionality at pressures three orders-of-magnitude larger results from the metamaterial vanes' unique features: (1) extremely low areal density $\left(0.1 \mathrm{mg} / \mathrm{cm}^{2}\right)$ that reduces the vane mass and hub friction force by almost 100 times; (2) high thermal resistivity that increases the cross-vane temperature difference; and (3) microchannels that enable through-vane thermal transpiration gas flows.

Each nanocardboard vane features a basketweave-style five-flow-channel pattern to amplify the thermal transpiration force. We manufactured these vanes using microfabrication techniques in four stages: (1) silicon mold creation using photolithography and reactive ion etching; (2) mold conformal coating using atomic layer deposition; (3) carbon nanotube drop-casting and oxygen plasma etching; and (4) mold cleaving and removing using $\mathrm{XeF}_{2}$ isotropic etching [1,5].

We measured the temperature and rotation speed of the radiometer using thermal and video cameras while illuminating it using an octagonal LED array. We found that our radiometer could operate at atmospheric pressure, and that its rotation rate increased with light intensity. To our knowledge, no other radiometers have achieved such functioning in ambient air. Lastly, we simulated the radiometer's fluid dynamics, obtaining similar trends between its rotation speed and light intensity and achieving order-of-magnitude agreement with our experiments.

Keywords: Crookes radiometer; microporous plate; photophoresis. 


\section{Introduction}
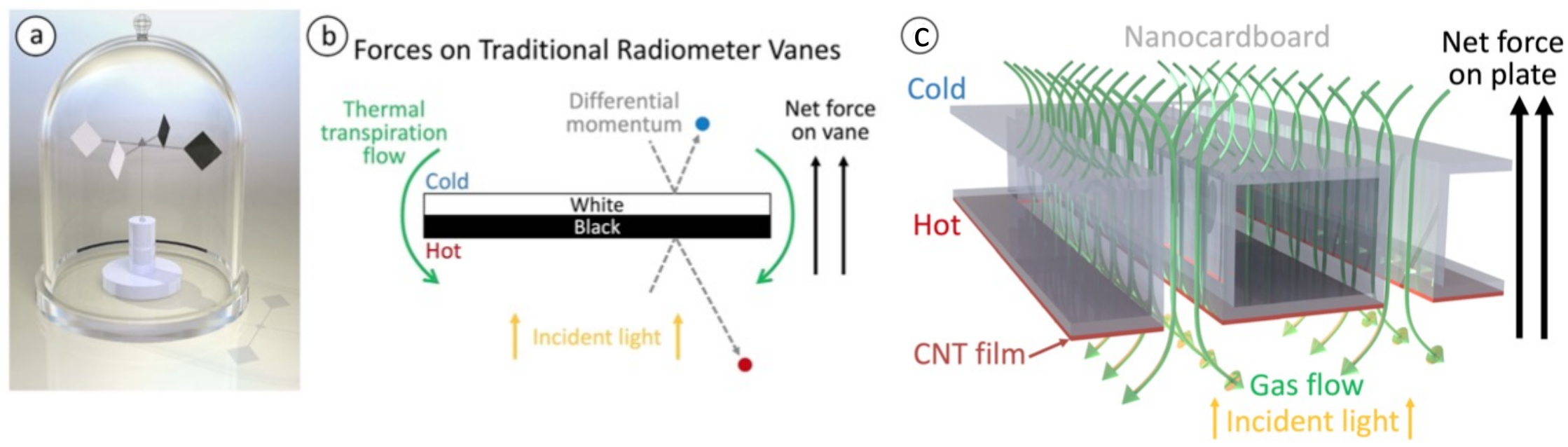

(a) Photorealistic rendering of a traditional radiometer in a vacuum jar. (b) Schematic diagram showing forces on traditional radiometer vanes. (c) Schematic diagram showing thermal creepinduced gas flow though nanocardboard channels. 


\section{Results and Discussion: Video}

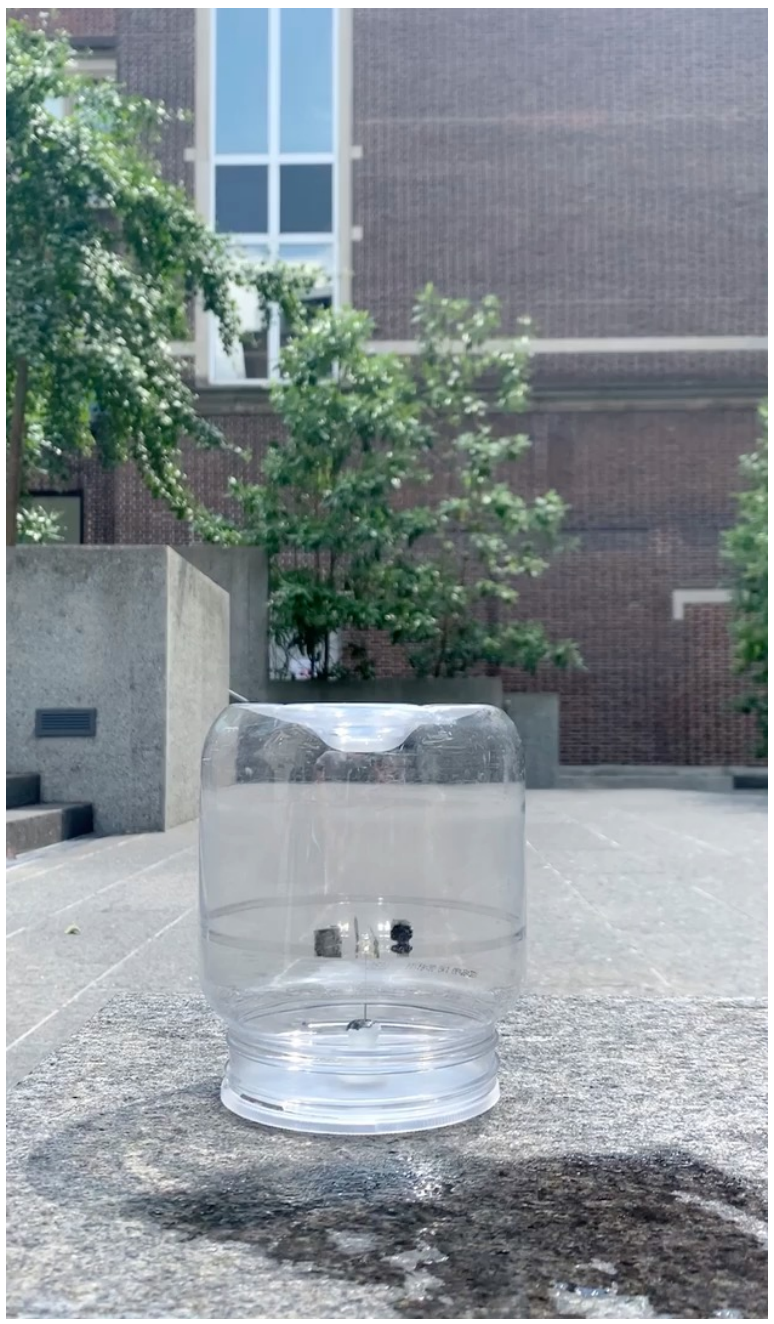

Video of radiometer operating under natural insolation $\left(1 \mathrm{~kW} / \mathrm{m}^{2}\right)$. 


\section{Results and Discussion: Fabrication}
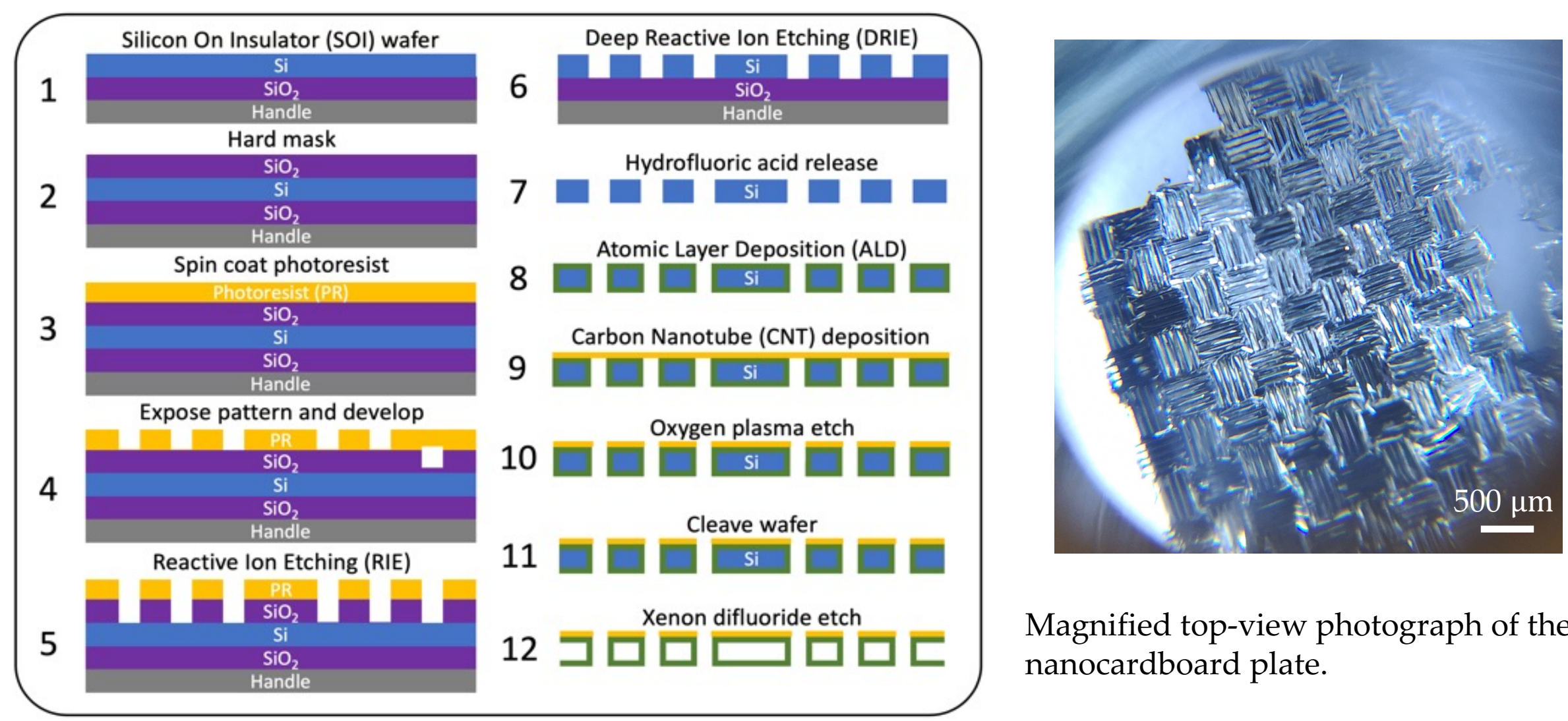

Magnified top-view photograph of the nanocardboard plate.

Vane fabrication process. 


\section{Results and Discussion: Setup}
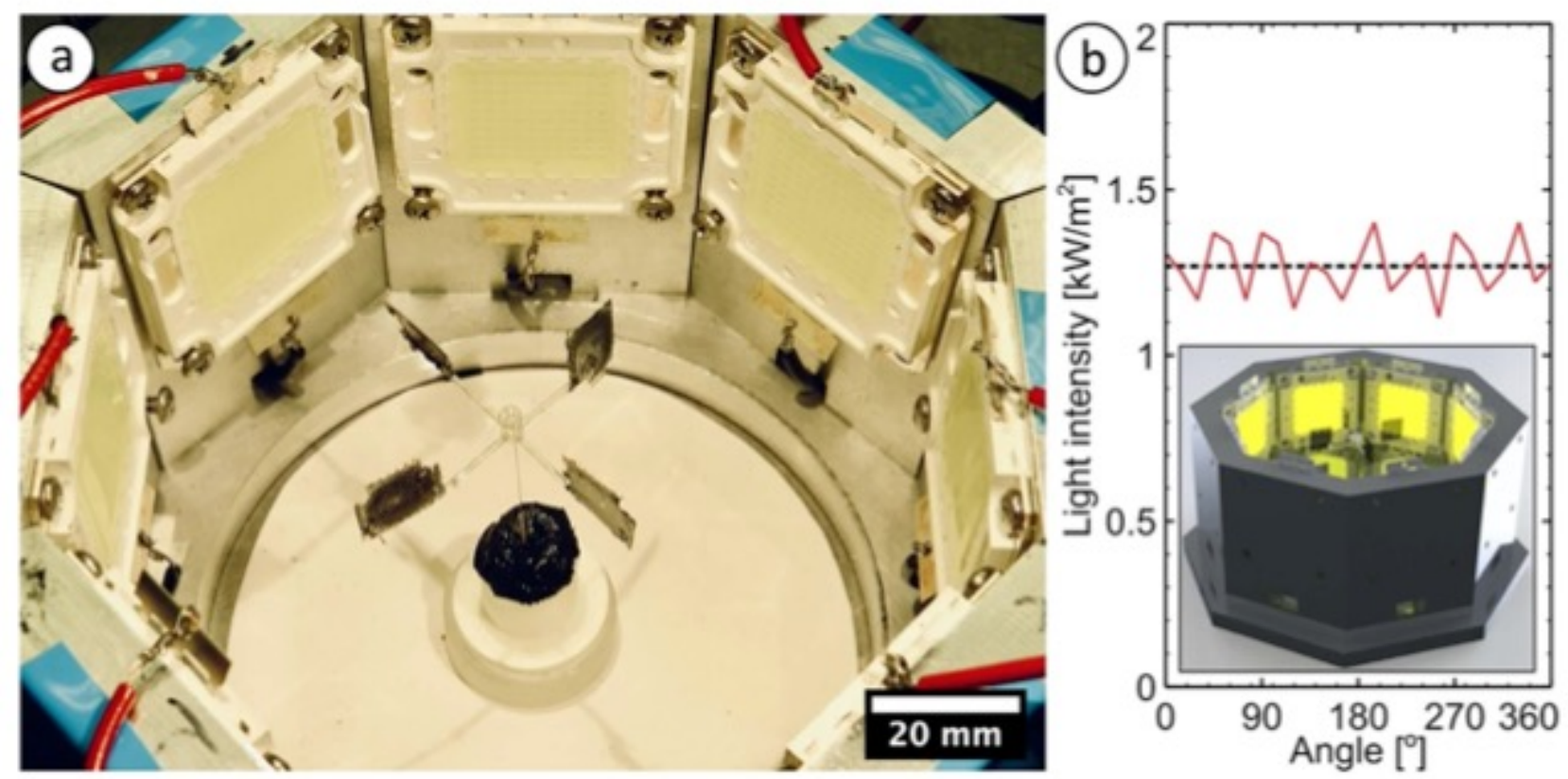

(a) Photograph of radiometer inside test chamber. (b) Light intensity as a function of angular position within the chamber at a radius of $13 \mathrm{~mm}$. Inset: Computer rendering of chamber showing LED illumination. 


\section{Results and Discussion: Measurement}

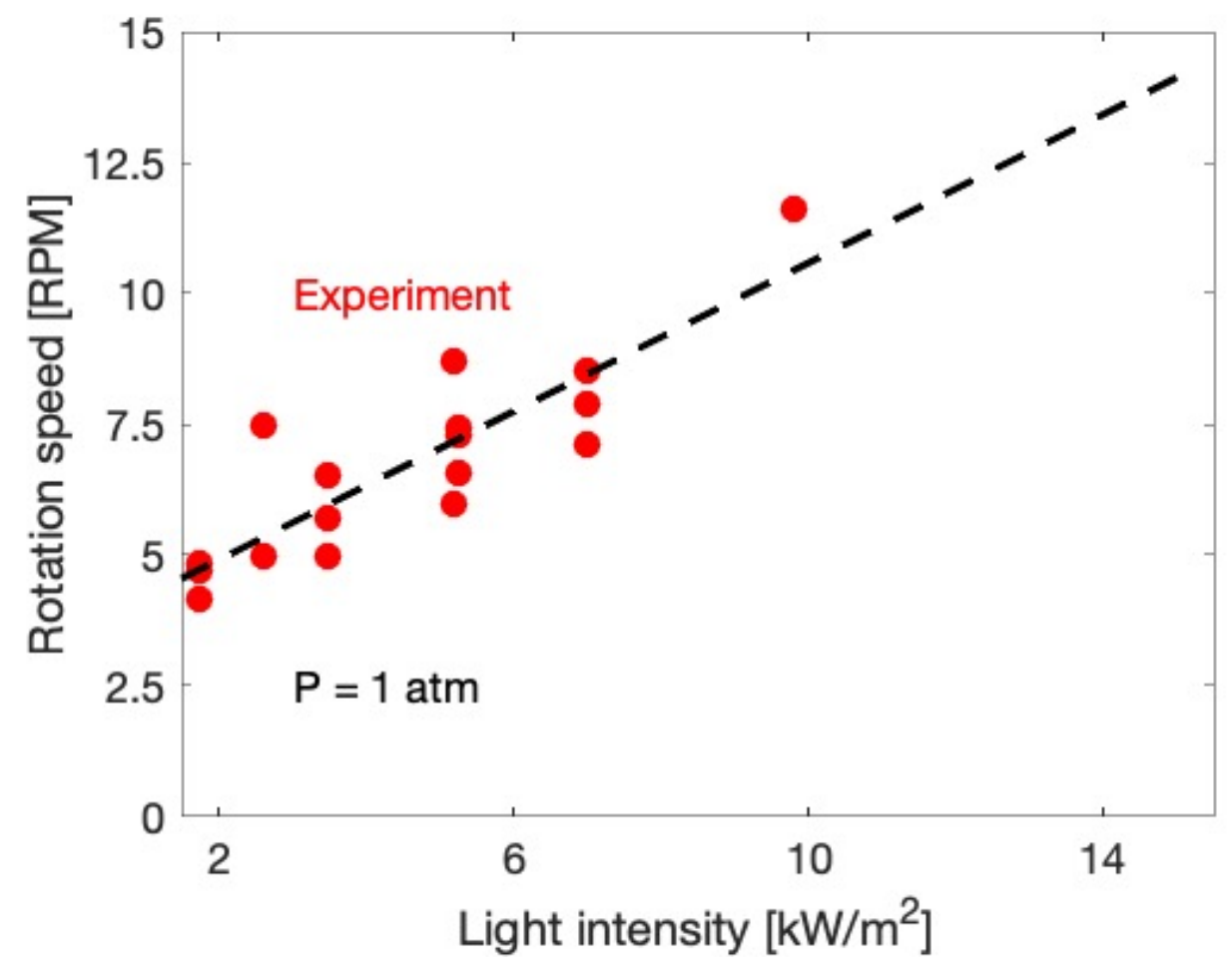

Experimentally-measured rotation speeds as a function of light intensity incident on the vanes.

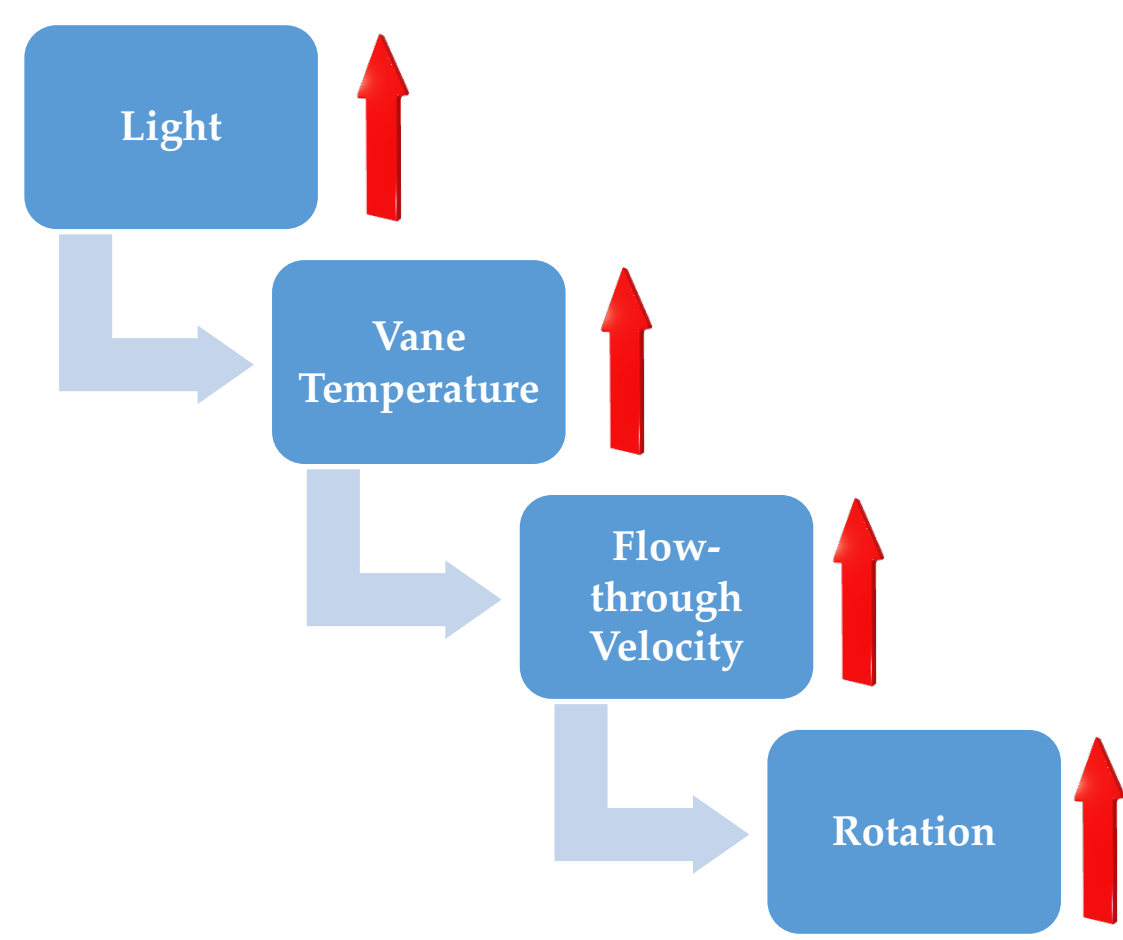

Explanation for the positive relationship between rotation speed and light intensity. 


\section{Results and Discussion: Simulation}

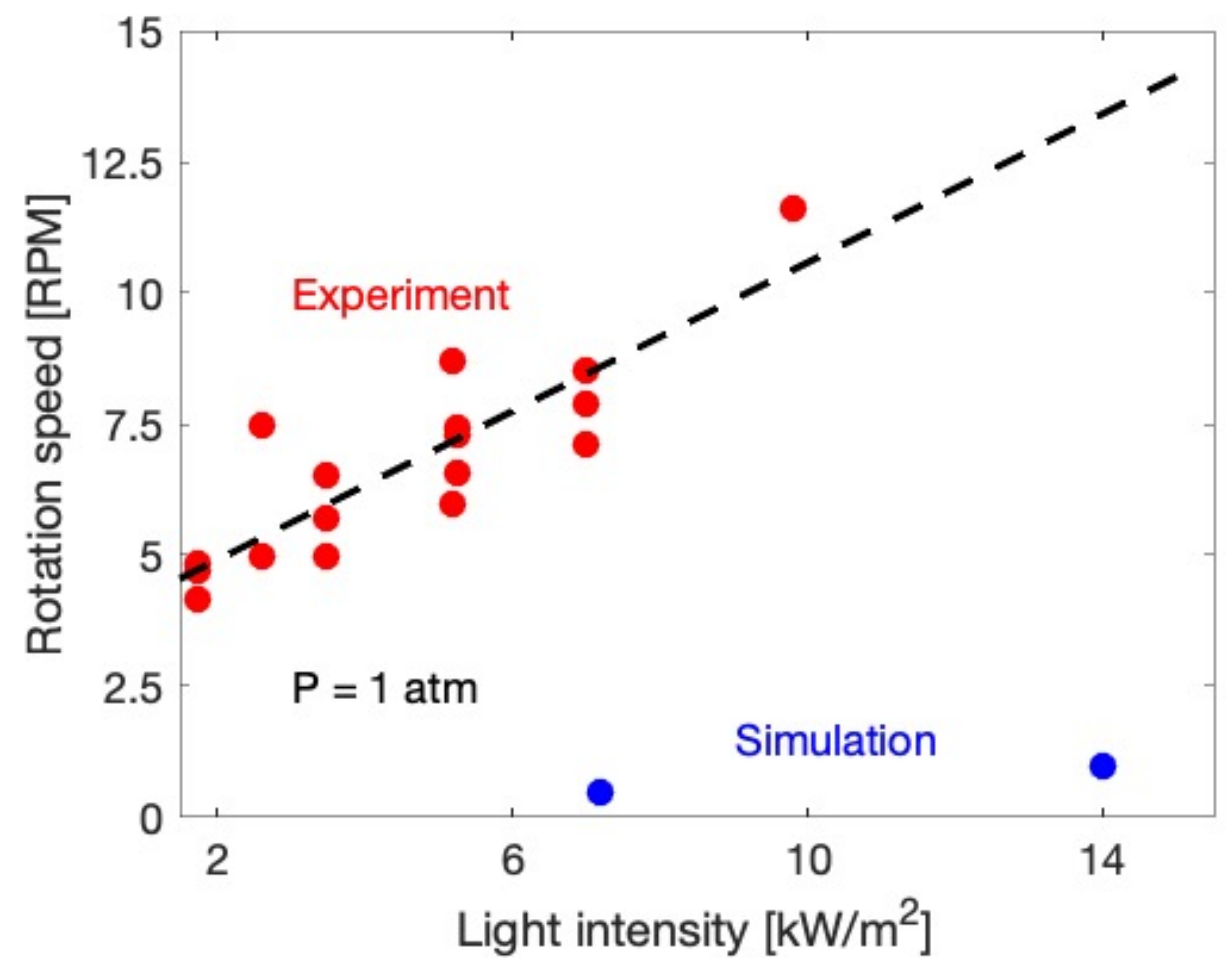

Experimentally-measured and simulated rotation speeds as a function of light intensity incident on the vanes.

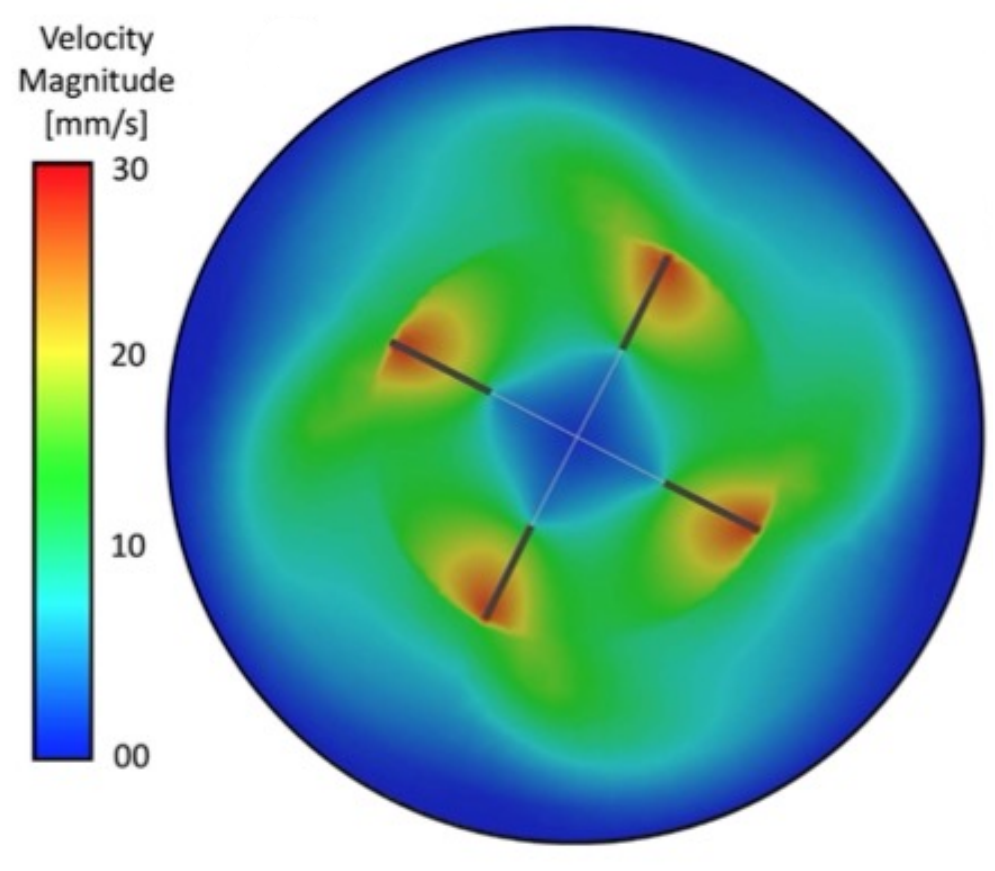

Top view of the flow field in an atmosphericpressure numerical simulation in which the $1.5-\mathrm{cm}$ square vanes rotated at $10 \mathrm{RPM}$. 


\section{Conclusions}

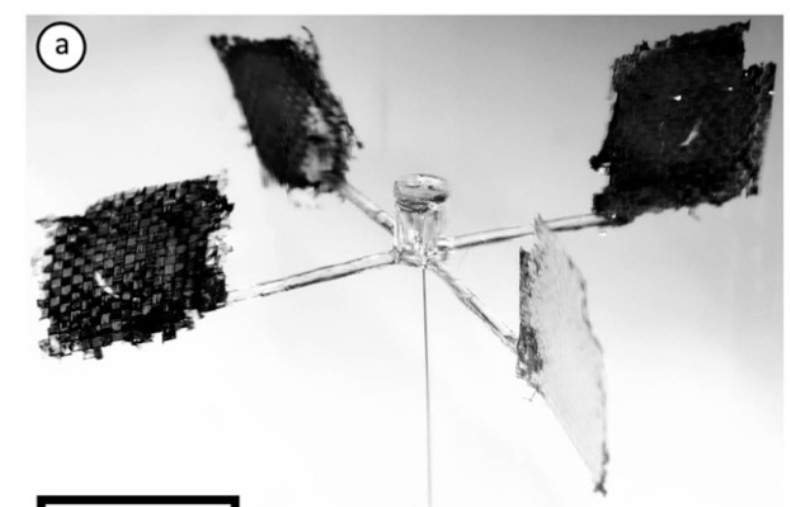

$10 \mathrm{~mm}$

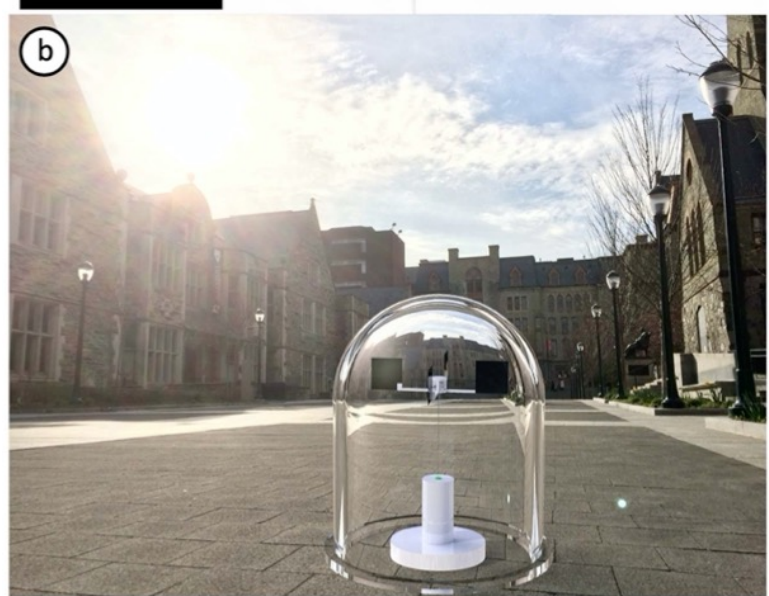

- First Crookes radiometer at $1 \mathrm{~atm}$;

- Nanocardboard architecture;

- Qualitative agreement with theory;

- Applications: microflyers, photogenerators, etc.

- Next step: smaller, pattern.

(a) Photograph of vanes glued to the hub. The vanes are shown prior to the $\mathrm{XeF}_{2}$ etch process. (b) Photorealistic rendering of the radiometer under sunlight on the University of Pennsylvania campus. 


\section{Acknowledgments}

The authors thank Prof. Howard Hu of the Department of Mechanical Engineering and Applied Mechanics, University of Pennsylvania, for his help with the simulations and the Staff of the Singh Center for Nanotechnology, Nanoscale Characterization Facility, and the Scanning and Local Probe facility at the same university.

\section{Thanks for watching!}




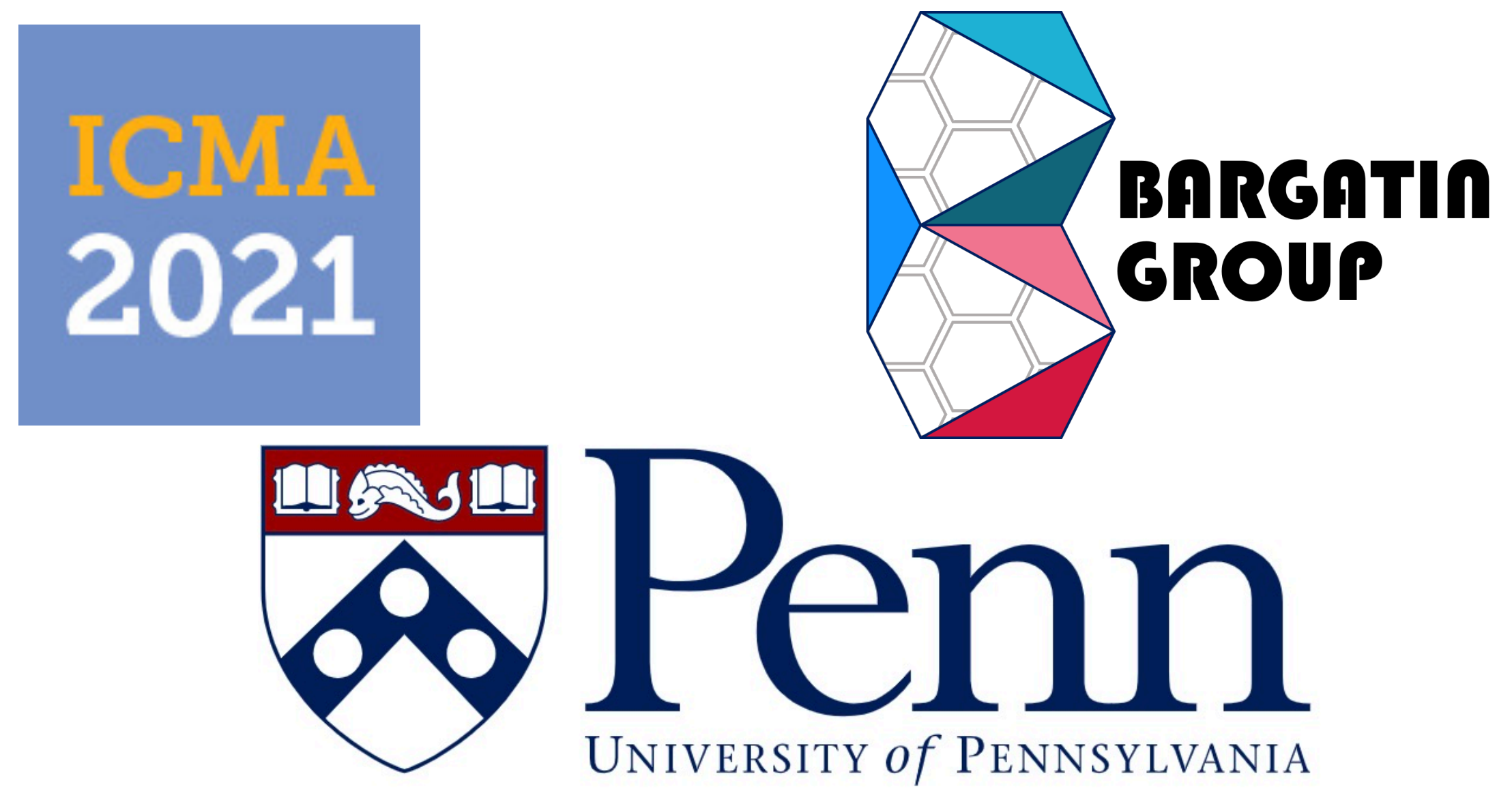

